\title{
Multi-Objective Fuzzy Chance Constrained Fuzzy Goal Programming for Capacitated Transportation Problem
}

\author{
A.K. Bhargava \\ Associate Professor, \\ Department of Mathematics, \\ M.M.H. College, Ghaziabad- \\ 201001 \\ Affiliated: C.C.S. University, \\ Meerut (U.P.), Pin-250001, \\ India
}

\author{
S.R. Singh \\ Associate Professor, \\ Department of Mathematics, \\ D.N. College, Meerut-250002 \\ Affiliated: C.C.S. University, \\ Meerut (U.P.), Pin-250001, \\ India
}

\author{
Divya Bansal \\ Research Scholar, Banasthali \\ University, Rajasthan, \\ Pin-304022, India
}

\begin{abstract}
This paper presents a multi-objective fuzzy chance constrained capacitated transportation problem based on fuzzy goal programming problem with capacity restrictions on commodities which are shipped from different sources to different destinations. The capacity of each origin and the demand of each destination are considered random in nature with fuzzy normal stochastic parameters following normal distribution with fuzzy mean and fuzzy variance respectively. These inequality constraints are also considered as fuzzy probabilistic in nature assuming to be triangular fuzzy numbers. Further, the supply and demand constraints are converted into equivalent deterministic forms. Then, we define the fuzzy goal tolerance limit of each of the objective functions which are then characterized by the associated membership functions. In the solution process, the fuzzy parameters are defuzzied by applying graded mean integration method which provided a satisfactory result. Further, an illustrative example is solved to demonstrate the effectiveness of the proposed model.
\end{abstract}

\section{Keywords}

Fuzzy goal programming, fuzzy chance constrained programming, capacitated transportation problem, multi objective decision making, graded mean integration method.

\section{INTRODUCTION}

Transportation problems involving single objective are a special type of linear programming (LP) problems with sources and destinations. Several quantitative techniques in Operations Research (OR) have been used for solving transportation problems. The objective functions coefficient represents transportation cost, delivery time, number of goods transported, unfulfilled demand, and many others. In operations research, transportation problems involve multiple and conflicting goals such as the cost minimization, balancing work among the plants, transportation fleets, and many others which are achieved using goal programming (GP) technique. Goal programming extends linear programming to problems which involve multiple objectives. Here, instead of maximizing or minimizing the objective function, the deviation between the desired goals is minimized according to the assigned priorities [1]. GP is regarded as the most widely used multi-criteria decision making technique [2]. The simplest TP model was developed by Hitchcock [3]. The stepping stone method was presented by Charnes and Cooper [4] for explaining linear programming calculation in transportation problem. The solid TP model was further proposed by Haley [5] with single objective. Interactive solutions was presented by Ringuest and Rinks [6] for solving linear multiobjective TP. Current and Min [7] designed TP networks for multi objectives, namely, taxonomy and annotation. The capacity restriction in single objective of solid TP was considered by Misra and Das [8, 9].

The main difference between fuzzy goal programming (FGP) and GP is that in GP there are definite aspiration values for each objective set by DM that he/she wishes to achieve, whereas in FGP aspiration levels are imprecise in nature. The use of fuzzy set theory in GP was first considered by Narasimhan [10, 11, 12], Hannan [13, 14, 15], Ignizio [16]. Rubin and Narsimhan [17] and Tiwari et al. [18, 19, 20] have investigated various aspects of decision problem using FGP. An extensive review of these papers is given by Tiwari et al. [18]. Chanas and Kuchta [21] presented a survey of fuzzy goal programming including three new approaches according to the role that fuzzy numbers play in them. Dantzig [22, 23] developed linear programming under uncertainty and also provided solution of two-stage linear programs with uncertainty called as the stochastic programming. Hassin and Zemel [24] gave the probabilistic analysis of the capacitated TP where an asymptotic condition was shown on the supplies and demands in order to assure a feasible solution to the problem. Bit et al. [25] studied the fuzzy programming approach to multi criteria decision making transportation problem where the right hand side parameters of the constraints and the coefficients in the objective functions are fuzzy numbers. The fuzzy programming approach to chance constrained multi objective TP was further studied by Bit et al. [26] where the parameters are considered as standard normal, log-normal, uniform random variables. Cen and Hsieh [27] proposed the graded mean integral value of a generalized fuzzy number.

Pramanik and Roy [28] studied fuzzy goal programming (FGP) approach for multi objective capacitated TP. Recently, Pramanik and Banerjee [29] presented chance constrained multi-objective capacitated transportation problem based on fuzzy goal programming. Fuzzy goal programming is applied to chance constrained multilevel programming problems [30]. Also, fuzzy goal programming approach on computation of the fuzzy arithmetic mean is proposed [31]. Further, Nayebi et al. [32] presented a fuzzy-chance constrained multi-objective programming applications for inventory control model involving a fuzzy chance parameter following normal distribution.

In this paper, supply and demand are considered as fuzzy normal stochastic parameters following normal distribution with fuzzy mean $\tilde{m}_{a_{i}}, \tilde{m}_{b j}$ and fuzzy variance $\tilde{\sigma}_{a_{i}}^{2}, \tilde{\sigma}_{b j}^{2}$ respectively, each representing triangular fuzzy numbers. The inequality constraints are also considered as fuzzy probabilistic in nature. Thus, the allowed minimum 
probability is also assumed to be a triangular fuzzy number as $\tilde{\alpha}_{i}, \tilde{\beta}_{j}$. Further, the supply and demand constraints are converted into equivalent deterministic forms. The fuzzy parameters are also defuzzied by applying graded mean integration method.

\section{MATHEMATICAL MODEL INVOLVING FUZZY CHANCE CONSTRAINED MULTI-OBJECTIVE CAPACITATED TRANSPORTATION PROBLEM (FCCMOCTP)}

Consider a transportation problem involving $\mathrm{m}$ sources (origins) $\mathrm{O}_{\mathrm{i}}(\mathrm{i}=1,2, \ldots, \mathrm{m})$ and $\mathrm{n}$ destinations $\mathrm{D}_{\mathrm{j}}(\mathrm{j}=1,2, \ldots$, $n)$. At each source $\mathrm{O}_{i}(i=1,2, \ldots, m)$, let $a_{i}$ be the amount of product to be transported from $i$ th origin to the $j$ th destination $D_{j}$ in order to satisfy the demand $b_{j}(j=1,2, \ldots, n)$. Here, $a_{i}$, $b_{j}$ are considered as random variables which are assumed to be triangular fuzzy numbers with known distribution. Also, under $k$-th criterion, there exists a penalty $\mathrm{c}_{\mathrm{ij}}{ }^{\mathrm{k}}$ associated with transporting one unit of product from source $\mathrm{O}_{\mathrm{i}}$ to destination $\mathrm{D}_{\mathrm{j}}$. In general, $\mathrm{c}_{\mathrm{ij}}{ }^{\mathrm{k}}$ denotes the transportation costs, delivery time, damage charges, underused capacity, etc. Let $x_{i j}$ represents the unknown quantity transported from $i$-th origin to $j$-th destination. There also exist certain limitations on the amount of resources allocated in different cells as per capacitated transportation problem. Let $\mathrm{t}_{\mathrm{ij}}$ be the maximum amount of quantity transported from i-th source to $j$-th destination i.e. $x_{i j}<t_{i j}$. This restriction is called the capacitated restriction on the route $i$ to $j$. Here, an unbalanced transportation problem is considered.

This section presents a model in fuzzy environment. Consider developing the model where both $a_{i}$ and $b_{j}$ are random variables. Here, $a_{i}$ and $b_{j}$ follow normal distribution with fuzzy mean $\tilde{m}_{a_{i}}, \tilde{m}_{b j}$ and fuzzy variance $\tilde{\sigma}_{a_{i}}^{2}, \tilde{\sigma}_{b j}^{2}$ respectively. The allowed minimum probability is also assumed to be a fuzzy numbers as $\tilde{\alpha}_{i}, \tilde{\beta}_{j}$. Then the above unbalanced TP can be defined as:

$\operatorname{Min} Z^{k}=\sum_{i=1}^{m} \sum_{j=1}^{n} c_{i j}^{k} x_{i j}, k=1,2, \ldots, K$

Subject to

$$
\begin{aligned}
& \operatorname{Pr} o b\left(\sum_{j=1}^{n} x_{i j} \leq \tilde{a}_{i}\right) \geq 1-\tilde{\alpha}_{i}, i=1,2, \ldots, m \\
& \operatorname{Pr} o b\left(\sum_{i=1}^{m} x_{i j} \geq \tilde{b}_{j}\right) \geq 1-\tilde{\beta}_{j}, j=1,2, \ldots, n \\
& 0 \leq x_{i j} \leq t_{i j}, i=1,2, \ldots, m, j=1,2, \ldots, n \\
& 0<\tilde{\alpha}_{i}<1 \\
& 0<\tilde{\beta}_{j}<1
\end{aligned}
$$

Here, $\alpha_{i}, \beta_{j}$ denotes the confidence levels for each constraint respectively.

The above fuzzy numbers are assumed to triangular fuzzy numbers parameterized by a triplet expressed in the form $\left(a_{1}, a_{2}, a_{3}\right)$ where $a_{1}$ and $a_{3}$ are the lower and upper limits of $a$ fuzzy number and $a_{2}$ is the pick value of that fuzzy number.

\subsection{Triangular Fuzzy Number (TFN)}

Let $\tilde{A}$ be the triangular fuzzy number represented by the triplet $\left(\mathrm{a}_{1}, \mathrm{a}_{2}, \mathrm{a}_{3}\right)$ which is defined by its continuous membership function $\mu_{\tilde{A}}(\mathrm{y}): \mathrm{Y} \rightarrow[0,1]$ given by

$\mu_{\tilde{A}}(\mathrm{y})=\left\{\begin{array}{cl}\frac{y-a_{1}}{a_{2}-a_{1}} & \text { if } a_{1} \leq y \leq a_{2} \\ 1 & \text { if } y=a_{2} \\ \frac{a_{3}-y}{a_{3}-a_{2}} & \text { if } a_{2} \leq y \leq a_{3} \\ 0 & \text { otherwise }\end{array}\right.$

The chance constraints are converted into equivalent deterministic constraints with the help of fuzzy mean, fuzzy variance and fuzzy confidence levels as described in the section below.

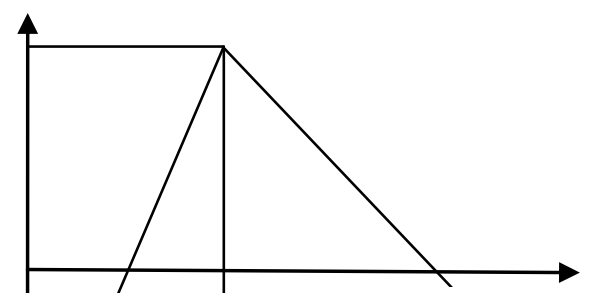

0

$$
a_{1} \quad y=a_{2}
$$

1: Triang _............... _.

\subsection{Deterministic Constraints Construction}

Consider the fuzzy chance constraints of the form:

$$
\operatorname{Pr} o b\left(\sum_{j=1}^{n} x_{i j} \leq \tilde{a}_{i}\right) \geq 1-\tilde{\alpha}_{i}, i=1,2, \ldots, m
$$

The above constraint can be rewritten in the form:

$$
\begin{aligned}
& \operatorname{Pr} o b\left(\frac{\sum_{j=1}^{n} x_{i j}-\tilde{m}_{a_{i}}}{\sqrt{\tilde{\sigma}_{a_{i}}^{2}}} \leq \frac{\tilde{a}_{i}-\tilde{m}_{a_{i}}}{\sqrt{\tilde{\sigma}_{a_{i}}^{2}}}\right) \geq 1-\tilde{\alpha}_{i}, i=1,2, \ldots, m \\
& \tilde{\alpha}_{i} \geq 1-\operatorname{Pr} o b\left(\frac{\sum_{j=1}^{n} x_{i j}-\tilde{m}_{a_{i}}}{\sqrt{\tilde{\sigma}_{a_{i}}^{2}}} \leq \frac{\tilde{a}_{i}-\tilde{m}_{a_{i}}}{\sqrt{\tilde{\sigma}_{a_{i}}^{2}}}\right), i=1,2, \ldots, m \\
& \tilde{\alpha}_{i} \geq \operatorname{Pr} o b\left(\frac{\sum_{j=1}^{n} x_{i j}-\tilde{m}_{a_{i}}}{\sqrt{\tilde{\sigma}_{a_{i}}^{2}}}>\frac{\tilde{a}_{i}-\tilde{m}_{a_{i}}}{\sqrt{\tilde{\sigma}_{a_{i}}^{2}}}\right), i=1,2, \ldots, m \\
& \rho^{-1}\left(\tilde{\alpha}_{i}\right) \geq \frac{\sum_{j=1}^{n} x_{i j}-\tilde{m}_{a_{i}}}{\sqrt{\tilde{\sigma}_{a_{i}}^{2}}}, i=1,2, \ldots, m \\
& \sum_{j=1}^{n} x_{i j} \leq \tilde{m}_{a_{i}}+\rho^{-1}\left(\tilde{\alpha}_{i}\right) \sqrt{\tilde{\sigma}_{a_{i}}^{2}}, i=1,2, \ldots, m
\end{aligned}
$$

where $\rho($.$) and \rho^{-1}($.$) represent the distribution function and$ inverse of distribution function of standard normal variable respectively.

Similarly, consider the second fuzzy chance constraint of the form: 
$\operatorname{Pr} o b\left(\sum_{i=1}^{m} x_{i j} \geq \tilde{b}_{j}\right) \geq 1-\tilde{\beta}_{j}, j=1,2, \ldots, n$

The above constraint can be rewritten in the form:

$\operatorname{Pr} o b\left(\frac{\sum_{j=1}^{n} x_{i j}-\tilde{m}_{b j}}{\sqrt{\tilde{\sigma}_{b j}^{2}}} \geq \frac{\tilde{b}_{j}-\tilde{m}_{b j}}{\sqrt{\tilde{\sigma}_{b j}^{2}}}\right) \geq 1-\tilde{\beta}_{j}, j=1,2, \ldots, n$

$\tilde{\beta}_{j} \leq 1-\operatorname{Pr} o b\left(\frac{\sum_{j=1}^{n} x_{i j}-\tilde{m}_{b j}}{\sqrt{\tilde{\sigma}_{b j}^{2}}} \geq \frac{\tilde{b}_{j}-\tilde{m}_{b j}}{\sqrt{\tilde{\sigma}_{b j}^{2}}}\right), j=1,2, \ldots, n$

$\rho\left(\frac{\sum_{j=1}^{n} x_{i j}-\tilde{m}_{b j}}{\sqrt{\tilde{\sigma}_{b j}^{2}}}\right) \geq 1-\tilde{\beta}_{j}, j=1,2, \ldots, n$

$1-\rho\left(-\frac{\sum_{j=1}^{n} x_{i j}-\tilde{m}_{b j}}{\sqrt{\tilde{\sigma}_{b j}^{2}}}\right) \geq 1-\tilde{\beta}_{j}, j=1,2, \ldots, n$

$\rho\left(-\frac{\sum_{j=1}^{n} x_{i j}-\tilde{m}_{b j}}{\sqrt{\tilde{\sigma}_{b j}^{2}}}\right) \leq \tilde{\beta}_{j}, j=1,2, \ldots, n$

$-\frac{\sum_{j=1}^{n} x_{i j}-\tilde{m}_{b j}}{\sqrt{\tilde{\sigma}_{b j}^{2}}} \leq \rho^{-1}\left(\tilde{\beta}_{j}\right), j=1,2, \ldots, n$

$\sum_{j=1}^{n} x_{i j} \geq \tilde{m}_{b j}-\rho^{-1}\left(\tilde{\beta}_{j}\right) \sqrt{\tilde{\sigma}_{b j}^{2}}, j=1,2, \ldots, n$

Equations (7) and (8) are determined at each of the triangular fuzzy numbers, $\tilde{m}_{a_{i}}, \tilde{m}_{b j}, \tilde{\sigma}_{a_{i}}^{2}, \tilde{\sigma}_{b j}^{2}$ and $\tilde{\alpha}_{i}, \tilde{\beta}_{j}$.

Then this model is reduced to deterministic multi-objective capacitated transportation problem defined as follows:

$\operatorname{Min} Z^{k}=\sum_{i=1}^{m} \sum_{j=1}^{n} c_{i j}^{k} x_{i j}, k=1,2, \ldots, K$

Subject to the constraints:

$\sum_{j=1}^{n} x_{i j} \leq \tilde{m}_{a_{i}}+\rho^{-1}\left(\tilde{\alpha}_{i}\right) \sqrt{\tilde{\sigma}_{a_{i}}^{2}}, i=1,2, \ldots, m$

$\sum_{j=1}^{n} x_{i j} \geq \tilde{m}_{b j}-\rho^{-1}\left(\tilde{\beta}_{j}\right) \sqrt{\tilde{\sigma}_{b j}^{2}}, j=1,2, \ldots, n$

$0 \leq x_{i j} \leq t_{i j}, i=1,2, \ldots, m, j=1,2, \ldots, n$

$0<\tilde{\alpha}_{i}<1$

$0<\tilde{\beta}_{j}<1$

\section{FGP MODEL FORMULATION OF}

\section{CCMOCTP}

Let $Z_{k}$ represents the objective function to minimize the transportation cost, time, damages during transportation subject to the system constraints (9), (10) and (11). Let $Z_{k}^{L}$ and $Z_{k}^{U}$ represents the individual best and worst (or upper and lower) solution of the objective function subject to system constraints respectively. The fuzzy goals are represented as:

$Z_{k} \wedge Z_{k}^{L}$.

The linear membership function for the fuzzy goal can be written
$\mu_{k}\left(Z_{k}\right)=\left\{\begin{array}{cc}1 & \text { if } Z_{k} \leq Z_{k}^{L} \\ \frac{Z_{k}^{U}-Z_{k}}{Z_{k}^{U}-Z_{k}^{L}} & \text { if } Z_{k}^{L} \leq Z_{k} \leq Z_{k}^{U} \\ 0 & \text { if } Z_{k} \geq Z_{k}^{U}\end{array}\right.$

proposed by Zimmermann [33].

Here $Z_{k}^{U}-Z_{k}^{L}$ is the tolerance range for the $k$-th goal.

Thus, the membership goal of each membership function using the model studied by Pramanik and Dey [34] can be written as:

$\mu_{k}\left(Z_{k}\right)+d_{k}^{-}=1$

Here, $d_{k}^{-}$represents the underachievement of the objective function.

Thus, the fuzzy goal programming for FCCMOCTP can be formulated as:

$\operatorname{Min} Z_{k}=\sum_{k=1}^{K} w_{k} d_{k}^{-}$

Subject to the constraints (9), (10), (11) and

$0 \leq d_{k}^{-} \leq 1$

Here,

$w_{k}=\frac{1}{Z_{k}^{U}-Z_{k}^{L}}$ is the weight associated with the k-th objective function.

As some of the parameters are assumed to be TFNs (Triangular Fuzzy Numbers), therefore, the problem involving fuzzy parameters is further defuzzied by applying graded mean integration method.

\subsection{Generalized Fuzzy Number}

The generalized fuzzy number $\tilde{A}$ with the membership function $\mu_{\tilde{A}}(\mathrm{y})$ (figure below) exhibit a fuzzy subset of the real line, where

$\mu_{\tilde{A}}(y)=\left\{\begin{array}{cl}L(y) & \text { if } a_{1} \leq y \leq a_{2} \\ 1 & \text { if } x=a_{2} \\ R(y) & \text { if } a_{2} \leq y \leq a_{3} \\ 0 & \text { otherwise }\end{array}\right.$

where $\mathrm{L}(\mathrm{y})$ and $\mathrm{R}(\mathrm{y})$ are continuous function of $\mathrm{y}$. Moreover, $\mathrm{L}(\mathrm{y})$ and $\mathrm{R}(\mathrm{y})$ are strictly monotonically increasing and strictly monotonically decreasing functions respectively, of $\mathrm{y}$ in $a_{1} \leq y \leq a_{2}$ and $a_{2} \leq y \leq a_{3}$.

\subsection{Graded Mean Integration value of Fuzzy Number}

According to Chen and Hsieh [27], the graded mean integral value of a generalized fuzzy number $\tilde{A}$ with the membership function $\mu_{\tilde{A}}(\mathrm{y})$ defined by $\mathrm{P}_{\mathrm{dGw}}(\tilde{A})$ of $\tilde{A}$ is given by 
$P_{d G w}(\tilde{A})=\frac{\int_{0}^{1} y\left\{(1-w) L^{-1}(y)+w R^{-1}(y)\right\} d y}{\int_{0}^{1} y d y}$

This implies

$$
P_{d G w}(\tilde{A})=2 \int_{0}^{1} y\left\{(1-w) L^{-1}(y)+w R^{-1}(y)\right\} d y
$$

where $w \in[0,1]$ is a pre-assigned parameter which represents the degree of optimism.

$\mathrm{w}=1$ indicates optimistic approach of decision maker's; $\mathrm{w}=$ 0 indicates pessimistic approach of decision maker's and $\mathrm{w}=$ 0.5 indicates moderate optimistic approach of decision maker's. The graded mean integration value of triangular fuzzy number.

$\tilde{A}=\left(a_{1}, a_{2}, a_{3}\right)$ with moderate optimistic approach of decision maker is given by

$$
P_{d G 0.5}(\tilde{A})=\frac{a_{1}+a_{2}+a_{3}}{6}
$$

Therefore, the above model where both $a_{i}$ and $b_{j}$ are random variables following normal distribution with fuzzy mean $\tilde{m}_{a_{i}}, \tilde{m}_{b j}$ and fuzzy variance $\tilde{\sigma}_{a}^{2}, \tilde{\sigma}_{b j}^{2}$ respectively, along with the minimum probability assuming fuzzy number as $\tilde{\alpha}_{i}, \tilde{\beta}_{j}$ which are TFNs are represented using graded mean integration value method with moderate optimistic.

\section{ILLUSTRATIVE EXAMPLE}

Consider the following example taken from Pramanik and Banerjee [29] to demonstrate the potentiality of the proposed FGP models. Consider three origins and three destinations. The TP cost, time and the damage charges during the transportation are represented by three square matrices of order three. The matrices are given bellow:

$\begin{array}{lccc} & 3 & 4 & 13 \\ \text { Cost matrix: } & 12 & 14 & 7 \\ & 15 & 10 & 8 \\ & 9 & 1 & 3 \\ \text { Time matrix: } & 2 & 4 & 6 \\ & 8 & 12 & 10 \\ & 8 & 9 & 11 \\ \text { Damage charge: } & 3 & 4 & 7 \\ & 2 & 1 & 6\end{array}$

Then the objective functions can be represented by

Min $\mathrm{Z}_{1}=\left(3 \mathrm{x}_{11}+4 \mathrm{x}_{12}+13 \mathrm{x}_{13}\right)+\left(12 \mathrm{x}_{21}+14 \mathrm{x}_{22}+7 \mathrm{x}_{23}\right)+$ $\left(15 x_{31}+10 x_{32}+8 x_{33}\right)$

Min $\mathrm{Z}_{2}=\left(9 \mathrm{x}_{11}+1 \mathrm{x}_{12}+3 \mathrm{x}_{13}\right)+\left(2 \mathrm{x}_{21}+4 \mathrm{x}_{22}+6 \mathrm{x}_{23}\right)+$ $\left(8 \mathrm{x}_{31}+12 \mathrm{x}_{32}+10 \mathrm{x}_{33}\right)$

Min $\mathrm{Z}_{3}=\left(8 \mathrm{x}_{11}+9 \mathrm{x}_{12}+11 \mathrm{x}_{13}\right)+\left(3 \mathrm{x}_{21}+4 \mathrm{x}_{22}+7 \mathrm{x}_{23}\right)+$ $\left(2 x_{31}+1 x_{32}+6 x_{33}\right)$

Subject to

$$
\begin{aligned}
& \operatorname{Pr} o b\left(\sum_{j=1}^{n} x_{i j} \leq \tilde{a}_{i}\right) \geq 1-\tilde{\alpha}_{i} ; i=1,2,3 \\
& \operatorname{Pr} o b\left(\sum_{i=1}^{m} x_{i j} \geq \tilde{b}_{j}\right) \geq 1-\tilde{\beta}_{j} ; j=1,2,3
\end{aligned}
$$

The capacitated constraints are given below:

$$
\begin{aligned}
& 0<\mathrm{x}_{11}<6,0<\mathrm{x}_{12}<7,0<\mathrm{x}_{13}<13 \\
& 0<\mathrm{x}_{21}<6,0<\mathrm{x}_{22}<2,0<\mathrm{x}_{23}<13 \\
& 0<\mathrm{x}_{31}<4,0<\mathrm{x}_{32}<7,0<\mathrm{x}_{33}<14 \\
& x_{13}+x_{23}+x_{33} \geq 21.17-\sqrt{16.33} \rho^{-1}(0.031) \\
& =21.17-\sqrt{16.33}(1.866)=13.63
\end{aligned}
$$

The triangular fuzzy mean, variance, and the confidence levels are described as:

$$
\begin{aligned}
& \tilde{m}_{a_{1}}=(11,12,14), \tilde{\sigma}_{a_{1}}^{2}=(8,9,11), \tilde{\alpha}_{1}=(0.005,0.01,0.015) \\
& \tilde{m}_{a_{2}}=(14,15,17), \tilde{\sigma}_{a_{2}}^{2}=(3,4,6), \tilde{\alpha}_{2}=(0.01,0.02,0.04) \\
& \tilde{m}_{a_{3}}=(19,20,23), \tilde{\sigma}_{a_{3}}^{2}=(6,7,9), \tilde{\alpha}_{3}=(0.025,0.03,0.04) \\
& \tilde{m}_{b_{1}}=(8,9,11), \tilde{\sigma}_{b_{1}}^{2}=(1.5,2,4), \tilde{\beta}_{1}=(0.005,0.01,0.015) \\
& \tilde{m}_{b_{2}}=(12,13,15), \tilde{\sigma}_{b_{2}}^{2}=(7,8,10), \tilde{\beta}_{2}=(0.01,0.02,0.04) \\
& \tilde{m}_{b_{3}}=(20,21,23), \tilde{\sigma}_{b_{3}}^{2}=(15,16,19), \tilde{\beta}_{3}=(0.025,0.03,0.04)
\end{aligned}
$$

The triangular fuzzy mean, variance, and the confidence levels are described as:

$$
\begin{aligned}
& \tilde{m}_{a_{1}}=\frac{11+4(12)+14}{6}=12.17, \tilde{\sigma}_{a_{1}}^{2}=\frac{8+4(9)+11}{6}=9.17 \\
& \tilde{\alpha}_{1}=\frac{0.005+4(0.01)+0.015}{6}=0.01 \\
& \tilde{\alpha}_{1}=\frac{0.005+4(0.01)+0.015}{6}=0.01
\end{aligned}
$$

Similarly, conducting the same for other, we get,

$$
\begin{aligned}
& \tilde{m}_{a_{2}}=15.17, \tilde{\sigma}_{a_{2}}^{2}=4.17, \tilde{\alpha}_{2}=0.022 \\
& \tilde{m}_{a_{3}}=20.33, \tilde{\sigma}_{a_{3}}^{2}=7.17, \tilde{\alpha}_{3}=0.031 \\
& \tilde{m}_{b_{1}}=9.17, \tilde{\sigma}_{b_{1}}^{2}=2.25, \tilde{\beta}_{1}=0.01 \\
& \tilde{m}_{b_{2}}=13.17, \tilde{\sigma}_{b_{2}}^{2}=8.17, \tilde{\beta}_{2}=0.022 \\
& \tilde{m}_{b_{3}}=21.17, \tilde{\sigma}_{b_{3}}^{2}=16.33, \tilde{\beta}_{3}=0.031
\end{aligned}
$$

Thus, the chance constraints (25) - (26) using the above values corresponding to the lower limit of the triangular fuzzy numbers and equation (9) and (10) can be represented as:

$$
\begin{aligned}
& x_{11}+x_{12}+x_{13} \leq 12.17+\sqrt{9.17} \rho^{-1}(0.01) \\
& =12.17+\sqrt{9.17}(2.326)=19.2136 \\
& x_{21}+x_{22}+x_{23} \leq 15.17+\sqrt{4.17} \rho^{-1}(0.022) \\
& =15.17+\sqrt{4.17}(2.014)=19.2827
\end{aligned}
$$


$x_{31}+x_{32}+x_{33} \leq 20.33+\sqrt{7.17} \rho^{-1}(0.031)$

$=20.33+\sqrt{7.17}(1.866)=25.33$

$x_{11}+x_{21}+x_{31} \geq 9.17-\sqrt{2.25} \rho^{-1}(0.01)$

$=9.17-\sqrt{2.25}(2.326)=5.681$

$x_{12}+x_{22}+x_{32} \geq 13.17-\sqrt{8.17} \rho^{-1}(0.022)$

$=13.17-\sqrt{8.17}(2.014)=7.413$

To determine the tolerance range of each objective, solve each objective separately for minimization and maximization of objective function with respect to the constraints obtained by using above methodology, that is, equations (27) and (30).

Thus, the tolerance ranges for the three objective functions are taken as:

[145, 614.83], [65.15, 443.78], [103.475, 409.77].

Thus the chance constraints fuzzy goal programming as described in (16), (17) and (18) can be formulated as:

Minimize

$\frac{1}{614.83-145} d_{1}^{-}+\frac{1}{443.78-65.15} d_{2}^{-}+\frac{1}{409.77-103.475} d_{3}^{-}$

Subject to

$\frac{614.83-Z_{1}}{614.83-145}+d_{1}^{-}=1$

$\frac{443.78-Z_{2}}{443.78-65.15}+d_{2}^{-}=1$

$\frac{409.77-Z_{3}}{409.77-103.475}+d_{3}^{-}=1$

Equations (27) and (30)

The capacitated constraints (27)

$0 \leq d_{i}^{-} \leq 1, i=1,2,3$

where $Z_{1}, Z_{2}, Z_{3}$ are same as specified in (22) - (24).

$\mu_{1}=\frac{614.83-Z_{1}}{614.83-145}$

$\mu_{2}=\frac{443.78-Z_{2}}{443.78-65.15}$

$\mu_{3}=\frac{409.77-Z_{3}}{409.77-103.475}$

Solving the above problem using LINGO, we obtain the following solution:

$\mathrm{x}_{11}=0, \mathrm{x}_{12}=7, \mathrm{x}_{13}=0, \mathrm{x}_{21}=5.68, \mathrm{x}_{22}=0.413, \mathrm{x}_{23}=13, \mathrm{x}_{31}=$ $0, x_{32}=0, x_{33}=0.63$.

The values of the membership functions are

$\mu_{1}=0.67, \mu_{2}=0.81, \mu_{3}=0.48$

and the values of the objective functions are obtained as:

$Z_{1}=197.99, Z_{2}=104.314, Z_{3}=176.475$

Thus we can conclude from the above results that graded mean integration method provide a satisfactory results.

\section{CONCLUSION}

This paper presents fuzzy chance constrained fuzzy goal programming and its application for solving CCMOCTP with fuzzy mean, fuzzy variance and fuzzy confidence level. Further, fuzzy parameters are defuzzied by applying graded mean integration method. The illustrative example shows that by defuzzification of triangular fuzzy numbers one can obtain a satisfactory solution.

In future this FGP model can be used in many practical problems like plant management, travelling salesman problems, resource allocation problem, assignment problems, etc. with crisp demands and supplies. The concept presented in this paper can also be applied for non-hierarchical as well as hierarchical organization involving multi-objective fractional programming problem.

\section{REFERENCES}

[1] Chowdary, B.V. and Slomp J. 2002. Production planning under dynamic product environment: A multi-objective goal programming approach, Research Report, University of Groningen.

[2] Sen, N. and Nandi M. 2012. A goal programming approach to rubber plantation planning in Tripura. Applied Mathematical Science 6 (124), 6171-6179.

[3] Hitchcock, F. L. 1941. Distribution of product from several sources to numerous localities. Journal of Mathematical Physics 20, 224-230.

[4] Charnes, A. and Cooper W. W. 1954. The stepping stone method for explaining linear programming calculation in transportation problem. Management Science 1, 49-69.

[5] Haley, K. B. 1963. The solid transportation problem. Operational Research 10, 448-463.

[6] Ringuest, J. L. and Rinks D. B. 1987. Interactive solutions for the linear multi-objective transportation problem. European Journal of Operational Research 32, 96-106.

[7] Current, J. and Min H. 1986. Multiobjective design of transportation networks: Taxonomy and annotation. European Journal of Operational Research 26, 187-201.

[8] Misra, S. and Das C. 1981a. Three-dimensional transportation problem with capacity restriction. New Zealand Operational Research 9, 47-58.

[9] Misra, S. and Das C. 1981b. Solid transportation problem with lower and upper bounds on the rim condition-A note. New Zealand Operational Research 9, 137-140.

[10] Narasimhan, R. 1980. Goal programming in a fuzzy environment. Decision Sciences 11, 325-336.

[11] Narasimhan, R. 1981. On fuzzy goal programmingSome comments. Decision Sciences 12, 532-538.

[12] Narasimhan, R. 1982. A geometric averaging procedure for constructing super transitive approximation to binary comparison matrices. Fuzzy Sets and Systems 8, 53-61.

[13] Hannan, E.L. 1981. Linear programming with multiple fuzzy goals. Fuzzy Sets and Systems 6, 235-248.

[14] Hannan, E.L. 1981. On fuzzy goal programming. Decision Sciences 12, 522-531. 
[15] Hannan, E.L. 1982. Contrasting fuzzy goal programming and fuzzy multicriteria programming. Decision Sciences $13,337-339$.

[16] Ignizio, J.P. 1982. On the (re) discovery of fuzzy goal programming. Decision Sciences 13, 331-336.

[17] Rubin, P.A. and Narasimhan R. 1984. Fuzzy goal programming with nested priorities. Fuzzy Sets and Systems 14, 115-129.

[18] Tiwari, R.N., Rao J. R. and Dharmar S. 1985. Some aspects of fuzzy goal programming, International Symposium on Mathematical Modeling of Ecological Environmental and Biological Systems, Kanpur, India.

[19] Tiwari, R.N., Dharmar S. and Rao J. R. 1987. Fuzzy goal programming-An additive model. Fuzzy Sets and Systems 24, 27-34.

[20] Tiwari, R.N., Dharmar S. and Rao J. R. 1986. Priority structure in fuzzy goal programming. Fuzzy Sets and Systems 19, 251-259.

[21] Chanas, S. and Kuchta D. 2002. Fuzzy goal programming-One notion, many meanings. Control and Cybernetics 31 (4), 871-890.

[22] Dantzig, G. B. and Mandansky A. 1961. On the solution of two-stage linear programs under uncertainty. In I. J. Neyman, Editor, Proc. 4th Berkeley Symp. Math. Stat. Prob., 165-176.

[23] Dantzig, G. B. 1955. Linear programming under uncertainty. Management Science 1, 197-206.

[24] Hassin, R. and Zemel E. 1988. Probabilistic analysis of the capacitated transportation problem. Mathematics of Operation Research 13, 80-89.

[25] Bit, A. K., Biswal M. P. and Alam S. S. 1992. Fuzzy programming approach to multi-criteria decision making transportation problem. Fuzzy Sets and Systems 50, 135141.
[26] Bit, A. K., Biswal M. P. and Alam S. S. 1994. Fuzzy programming approach to chance constrained multiobjective transportation problem. The Journal of Fuzzy Mathematics 2, 117-130.

[27] Chen, H. and Hsieh C.H. 1999. Graded mean integration representation of generalized fuzzy numbers. Journal of Chinese Fuzzy System Association 5 (2), 1-7.

[28] Pramanik, S. and Roy T. R. 2005. A fuzzy goal programming approach for multi-objective capacitated transportation problem. Tamsui Oxford Journal of Management Sciences 21, 75-88.

[29] Pramanik, S. and Banerjee D. 2012. Multi-objective chance constrained capacitated transportation problem based on fuzzy goal programming. International Journal of Computer Applications (0975-8887) 44 (20), 42-46.

[30] Kumar, M. and Pal B. B. 2013. Fuzzy goal programming to chance constrained multilevel programming problems. International Journal of Advanced Computer Research 3 (1), 193-200.

[31] Gunes, M. and Umarosman N. 2005. Fuzzy goal programming approach on computation of the fuzzy arithmetic mean. Mathematical and Computational Applications 10 (2), 221-220.

[32] Nayebi, M. A., Sharifi M., Shahriari M. R. and Zarabadipour O. 2012. Fuzzy-chance constrained multiobjective programming applications for inventory control model. Applied Mathematical Sciences 6 (5), 209-228.

[33] Zimmermann, H.-J. 1978. Fuzzy programming and linear programming with several objective functions. Fuzzy Sets and Systems 1, 45-55.

[34] Pramanik, S. and Dey P. P. 2011. Quadratic bi-level programming problem based on fuzzy goal programming approach. International Journal of Software Engineering and Applications 2 (4), 41-59.

[35] Scharge, L. 2013. Lingo 11.0. Lindo Systems Inc. Website: What's Best! http://www.lindo.com/.

[36] 